\section{Asthma im Erwachsenenalter in Abhängigkeit vom sozialen Umfeld}

Huovinen et al. vertreten die Ansicht, dass genetische Einflüsse alleine nicht die Zunahme des Asthmas in den letzten Dekaden erklären, vielmehr anderweitige, das Krankheitsbild prägende Einflüsse vorhanden sein müssten.

D ieser Frage gingen sie in einer Zwillingsstudie nach: Aus dem finnischen Zwillingsregister, welches gleichgeschlechtliche mono- und dizygote Zwillingspaare enthält, wählten sie 262 Paare im Alter von 20 bis 50 Jahren aus, die 1977, zu Beginn der Studie, keine Zeichen eines Asthma bronchiale boten. Die weitere Krankheitsentwicklung wurde bis 1993 verfolgt.

Die Diagnose eines Asthmas wurde durch ambulante oder stationäre fachärztliche Untersuchung gestellt, weiterhin gesichert durch Kenntnis der Verordnung asthmaspezifischer Medika-

\section{Klaus-Kalveram-Preis}

Zum vierten Mal wird der 1995 gestiftete Klaus-Kalveram-Preis verliehen. Dem Stiftungsgedanken entsprechend dient er der Förderung von Nachwuchswissenschaftlern in der Allergologie, speziell im Bereich der Arzneimittel- und Nahrungsmittelallergien und ist mit DM 5.000 dotiert.

Eingereicht werden können Arbeiten, die sich mit dem Themengebiet der Arznei- und Nahrungsmittelallergien wissenschaftlich beschäftigen und in sich abgeschlossen sind. Sie können auch bereits publiziert, sollten dann aber nicht älter als zwei Jahre sein.

Die Bewerber werden gebeten, ihre Arbeiten in deutscher oder englischer Sprache bis zum 1. August 2002 beim Vorsitzenden der Klaus-Kalveram-Stiftung Herrn Prof. Dr. Günther Forck, Coesfeldweg 57, D-48161 Münster, in 3-facher Ausführung einzureichen. Anfragen sind ebenfalls an diese Adresse zu richten.

Die Arbeit muss ebenso wie das beigefügte Autorenreferat ohne Hinweis auf den Autor sein. Name und Anschrift des Bewerbers bitte in einem verschlossenen Umschlag dem Manuskript beifügen.

Die Verleihung des Preises erfolgt anlässlich des 27. ÄDA-Kongresses, 13.-15. September 2002, in Dresden. mente, welche die Datenbank der finnischen Krankenversicherung ermöglichte. Eine Atopie wurde ebenfalls ärztlicherseits gesichert. Sozialer Status, Ausbildung usw. wurden ebenso wie der Grad der körperlichen Betätigung, Zigarettenkonsum und Tierhaltung mittels Fragebogen ermittelt.

Es zeigte sich, dass der atopische Zwilling ein erhöhtes Risiko hat, an Asthma zu erkranken. Der besser ausgebildete Zwilling hatte ein signifikant verringertes Risiko an Asthma zu erkranken, desgleichen der mit regelmäßiger körperlicher Betätigung. Ohne statistisch nach- er Arbeit zugrunde lag die statistische Auswertung eines an mehr als 10.000 finnische Studenten verschickten detaillierten Fragebogens.

Im Ergebnis fand sich eine statistisch signifikant erhöhte Asthmarate bei Studenten aus feuchten und/oder schimmeligen Wohnungen. Auch litten Atopiker aus solchen Wohnungen häufiger an Rhinitis und Neurodermitis als ihre vergleichbaren Kommilitonen in trockenen Wohnungen. Feuchte Wohnungen erhöhten daneben auch die Zahl der unspezischen Infekte.

Die Autoren führen diese Befunde im Wesentlichen auf die vermehrte Schimmelbildung und den erhöhten Milbenbestand in feuchten Wohnungen mit entsprechend erhöhter Sensibilisierungsrate zurück. Möglicherweise schädigen Sporen und flüchtige Ausdünstungen von Schimmelpilzen die hyperreagiblen Schleimhäute der allergiekranken Studenten zusätzlich. weisbaren Einfluss auf die Entwicklung eines Asthmas waren die Berufsausübung und die Tierhaltung. Schädlicher Einfluss des Zigarettenrauchens war mit Signifikanz nur bei Frauen nachweisbar.

\section{Fazit}

Für die Praxis ergibt sich aus dieser Studie die Konsequenz, im ärztlichen Gespräch die Eltern allergischer, speziell asthmatischer Kinder auf die Dringlichkeit einer möglichst qualifizierten Ausbildung hinzuweisen. Therapeutisch sollte der Asthmatiker in die Lage versetzt und gleichzeitig motiviert werden, regelmäßig eine körperliche Betätigung auszuüben. Selbstverständlich ist von allergiegefährdeten Berufen abzuraten.

\section{Dr. D. Bruchhausen, Wuppertal}

Huovinen E et al. Social predictors of adult asthma: a co-twin case-control study. Thorax 2001; 56: 234-6

\title{
Feuchte Wohnungen fördern Allergien und Infekte
}

Kilpelainen et al. untersuchten den Einfluss von Schimmel und Feuchtigkeit in Wohnungen auf Rhinitis und Konjunktivitis, Asthma, atopisches Ekzem sowie auf unspezifische „grippale“ Infekte.

Fazit

Der seit Jahren vermutete ungünstige Einfluss feuchter und verschimmelter Wohnungen auf allergische Erkrankungen wird eindeutig bestätigt. Allergiker sollten daher immer nach der Qualität ihrer Wohnung befragt werden. Gegebenenfalls ist eine sachgemäße Sanierung zu empfehlen.

Dr. D. Bruchhausen, Wuppertal

Kilpelainen $\boldsymbol{M}$ et al. Home dampness, current allergic diseases, and respiratory infections among young adults. Thorax 2001; 56: 462-7

Impressum „Der ÄDA informiert“

Verantwortlich für den Inhalt: Prof. Dr. Ludger Klimek, Wiesbaden ÄDA-Geschäftsstelle: ServiceSystems, Dreieich; Carin Fresle, Ursula Raab E-mail: aeda@sersys.de 